Check for updates

Cite this: Chem. Sci., 2018, 9, 6509

๑ All publication charges for this article have been paid for by the Royal Society of Chemistry

Received 25th April 2018

Accepted 27th June 2018

DOI: $10.1039 / \mathrm{c} 8 \mathrm{sc} 01889 \mathrm{~h}$

rsc.li/chemical-science

\section{Size-dependent rate acceleration in the silylation of secondary alcohols: the bigger the faster}

\author{
Marta Marin-Luna, (D) Benjamin Pölloth, (D) Fabian Zott (D) and Hendrik Zipse (D)*
}

Relative rates for the reaction of secondary alcohols carrying large aromatic moieties with silyl chlorides carrying equally large substituents have been determined in organic solvents. Introducing thoroughly matching pairs of big dispersion energy donor (DED) groups enhanced rate constants up to four times, notably depending on the hydrogen bond donor ability of the solvent. A linear correlation between computed dispersion energy contributions to the stability of the silyl ether products and experimental relative rate constants was found. These results indicate a cooperation between solvophobic effects and DED-groups in the kinetic control of silylation reactions.

\section{Introduction}

Aromatic interactions ${ }^{\mathbf{1}}$ play a central role in diverse fields such as organic synthesis, ${ }^{2}$ supramolecular self-assembly, ${ }^{3-5}$ molecular recognition ${ }^{6}$ or protein and peptide structures. ${ }^{7}$ They mainly result from the sum of three terms: ${ }^{\mathbf{8 9}}$ (1) an electrostatic component due to the electronic nature of the substituents at the interacting surfaces, ${ }^{10}(2)$ London dispersion interaction ${ }^{\mathbf{1 1 - 1 4}}$ as the attractive component of van der Waals forces, which arise due to interactions between induced dipoles, ${ }^{15}$ and (3) the solvophobic or hydrophobic effect, which results from a balance between solvent-solvent and solvent-solute interactions. ${ }^{16-18}$ Whereas numerous studies have detailed the nature of the electrostatic component, ${ }^{19-24}$ it is still a challenge to quantify individual dispersive and solvophobic effects in solution. ${ }^{25}$ Recent studies by Cockroft et al. ${ }^{26-29}$ and Shimizu et al. ${ }^{30,31}$ employ torsional molecular balances ${ }^{32}$ to measure these effects through the quantification of conformational equilibria. Most of the studies conclude that the dispersive interactions are of minor importance in solution ${ }^{33}$ and that the major contribution to the stabilization of the folded state results from the balance of solvent-solvent and solute-solvent interactions. The conceptually similar idea of using sizeable (rigid) dispersion energy donor groups (DED-group) in reagents and/or ligands in the development of stereoselective catalytic processes has also been explored recently, ${ }^{2,34}$ where it has been found that the appropriate placement of interacting DED-moieties in a system can lead to higher selectivity. Interactions between DED groups in bimolecular (associative) reactions were recently analysed for

Department of Chemistry, LMU München, Butenandtstrasse 5-13, 81377, München, Germany.E-mail: zipse@cup.uni-muenchen.de

$\dagger$ Electronic supplementary information (ESI) available: Protocol for competition experiments, experimental procedures, characterization data, NMR spectra, computational data. CCDC 1839390 and 1839391. For ESI and crystallographic data in CIF or other electronic format see DOI: $10.1039 / \mathrm{c} 8 \mathrm{sc} 01889 \mathrm{~h}$ acylation reactions of alcohols. ${ }^{34,36}$ For this latter class of reactions we have found that acylations mediated by TCAP (9-azajulolidine, marked in red in Fig. 1a) are fastest for pyrenylsubstituted secondary alcohols (marked in green Fig. 1) as compared to alcohols carrying smaller aromatic or even aliphatic substituents. ${ }^{35}$ In contrast, reaction rates hardly vary for acylation reagents of different size, which can most easily be accommodated in the general transition state structure shown in Fig. 1a. In the following we explore the question whether the silylation of secondary alcohols with silyl chloride reagents can be accelerated in a similarly targeted fashion through the use of sufficiently large DED substituents in the reagents and substrates. The silylation of alcohols is of outstanding importance in protection group strategies for the synthesis of complex molecular targets, ${ }^{37,38}$ and any extension of the currently available toolset will obviously be helpful for organic synthesis in general. The base-catalysed silylation of alcohols is commonly assumed to follow a Lewis base- rather than a general basecatalysed mechanism. ${ }^{3-42}$ In contrast to acylation reactions,

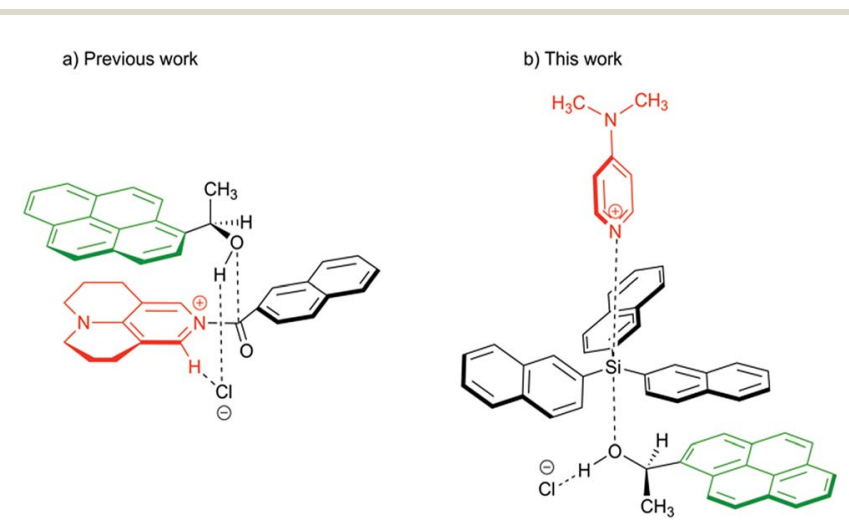

Fig. 1 Proposed transition structures for catalysed acylation (left) and silylation reaction (right) of 1-(1-pyrenyl)ethanol. 
silyl group transfer reactions proceed along an $\mathrm{S}_{\mathrm{N}}$ 2-like pathway, which implies the relative orientation of catalyst, reagent and substrate shown in the transition state cartoon in Fig. 1b. For this type of transition state structure, stabilizing interactions between appropriately placed DED substituents are expected between the alcohol and the reagent, but not between
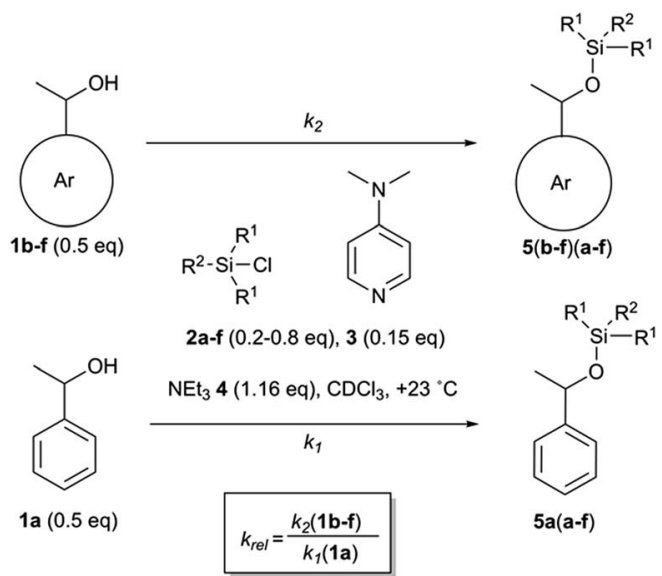

Scheme 1 Competition experiments between alcohol $1 \mathrm{a}$ and $1 \mathrm{~b}-\mathrm{f}$ with silyl reagents $2 a-f$. the alcohol and the catalyst. In order to probe this hypothesis, we have studied the reaction rates for the reaction of secondary alcohols with silyl chloride reagents carrying alkyl and aryl substituents of varying size. In addition, the influence of reaction temperature and solvent on the reaction rate was studied. The thermochemical stability of the products was explored by theoretical methods in an effort to quantify the dispersion energy contribution to the overall reaction driving force.

\section{Results and discussion}

Relative rate constants $k_{\text {rel }}$ for the Lewis base-catalysed silylation of alcohols 1a-1f with silyl chlorides $\mathbf{2 a - 2 f}$ were determined in $1: 1$ competition experiments employing 1-phenylethanol (1a) as the reference system (Scheme 1 and Fig. 2). The other substrate alcohols derived from 1a through annulation of one (as in $\mathbf{1 b}$ and 1c), two (as in 1d and 1e) or three (as in 1f) benzene rings to its phenyl group. Depending on the particular position of annulation, this generates no additional repulsive 1,5-interaction with the alcohol oxygen atom (as in 1c and 1e), one additional 1,5-interaction (as in $\mathbf{1 b}$ and $\mathbf{1 f}$ ), or two such interactions in 1d. The peri positions responsible for the repulsive 1,5-interactions are marked by grey circles in Fig. 2. The silyl chloride reagents chosen here grow in size from trimethylsilyl

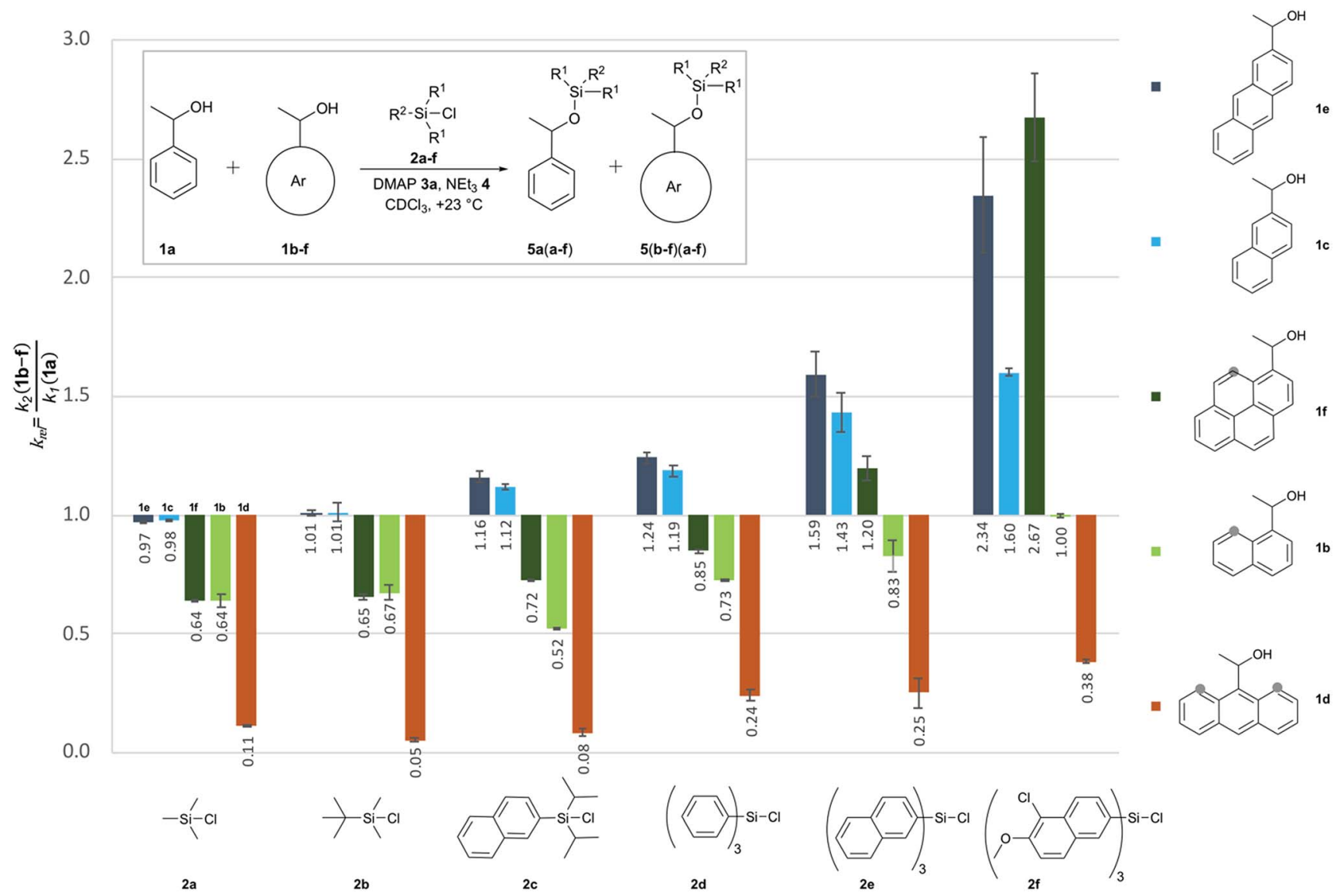

Fig. 2 Relative rate constants $\left(k_{\text {rel }}\right)$ for competition experiments between reference alcohol $1 \mathrm{a}$ and selected secondary alcohols $1 \mathrm{~b}-1 \mathrm{f}$ with silyl chlorides $2 a-f$ 
chloride 2a to substituted trinaphthylsilyl chloride $2 \mathbf{f}$ (see Fig. 2 and S1 of the ESI $\dagger$ ). The competition experiments described in Scheme 1 involve equal amounts of reference alcohol 1a and of one of the substrate alcohols $\mathbf{1 b}-\mathbf{f}$, a quantity of one of the silyl chlorides 2 sufficient enough to obtain between $20-80 \%$ turnover of the substrate alcohols 1 , a catalytic amount ( 0.15 eq. relative to alcohols $\mathbf{1}$ ) of $N, N$-dimethylaminopyridine (3, DMAP), and triethylamine (4) as the auxiliary base, in deuterated chloroform at a constant temperature of $+23{ }^{\circ} \mathrm{C}$. The relative rate constant $k_{\text {rel }}$ defined as the ratio of effective rate constants $k_{2}$ $(\mathbf{1 b}-\mathbf{f})$ over $k_{1}(\mathbf{1 a})$ was used as main control parameter and obtained from the mole distribution of reactants and products after completion of the reaction as determined by ${ }^{1} \mathrm{H}$ NMR spectroscopy (for full details see ESI $\dagger$ ). The resulting rate constant values are shown in Fig. 2 and in Tables S1-S5 of the ESI. $\dagger$

Relative reaction rates for the small trimethylsilyl chloride 2a (TMSCl) reagent show no response to the size of the alcohol $\pi$ systems, but are sensitive to the number of repulsive 1,5 -interactions. Reaction rates are therefore quite similar for alcohols 1a, 1c and 1e, then drop notably for alcohols with one 1,5interaction (1) and $\mathbf{1 f}$ ), and drop more strongly for the most hindered alcohol 1d, which reacts nine times slower than 1a. Moving to tert-butyldimethylsilyl chloride 2b (TBDMSCl) as a sterically more hindered and overall larger reagent we find practically no change in relative rate constants $k_{\text {rel }}$, except for the most hindered alcohol 1d, whose reactivity drops by another factor of two. With the results for smaller silyl chloride reagents in hand, we next investigated symmetrical silyl reagents $\mathbf{2 d - 2 f}$ containing sizeable aromatic substituents.

Reactions with triphenylsilyl chloride (2d, TPSCl) differ from those with TMSCl in two key aspects. First, all $k_{\text {rel }}$ values for silyl chloride $\mathbf{2 d}$ are larger than those for TMSCl (2a), the sterically unhindered alcohols 1c and 1e now reacting even faster than the reference alcohol 1a. Second, silyl chloride 2d differentiates more strongly between alcohols of different size, but identical degree of steric hindrance. Reaction rates for alcohols $\mathbf{1 b}$ and $\mathbf{1 f}$, for example, are quite similar for TMSCl (2a) and also for TBSCl (2b), but differ systematically for triphenylsilyl chloride (2d) in that the larger alcohol $\mathbf{1 f}\left(k_{\text {rel }}=0.85\right)$ reacts faster than alcohol $\mathbf{1 b}\left(k_{\mathrm{rel}}=0.73\right)$. Both factors can be seen at work in an enhanced way in reactions of the even larger trinaphthylsilyl chloride $\mathbf{2 e}$, where the sterically hindered, but pyrenyl-substituted alcohol $\mathbf{1 f}$ now reacts faster than the unhindered reference alcohol 1a $\left(k_{\text {rel }}\right.$ $=1.20$ ). Polar substituents were then added to the 5,6-positions of the naphthyl groups in silyl chloride $2 \mathbf{e}$ in order to increase its overall polarizability and the contact surface with alcohol reagents. Relative reaction rates for the resulting silyl chloride $2 \mathbf{f}\left(\mathrm{TN}^{*} \mathrm{SCl}\right)$ are all significantly larger than those for trinaphthylsilyl chloride 2e and appear to be mainly dominated by the size of the alcohol $\pi$-system. This makes pyrenyl-substituted alcohol $\mathbf{1 f}$ the most reactive substrate, closely followed by the less hindered anthracenyl-substituted alcohol 1e. A final test was performed with diisopropylnaphthylsilyl chloride 2c (DINSCl), which combines a single naphthyl with two $\alpha$ branched isopropyl substituents. The results obtained for this reagent are basically those for trinaphthylsilyl chloride $\mathbf{2 e}$, but
Table 1 Relative rate constants $\left(k_{\text {rel }}\right)$ for competition experiments between alcohol $1 \mathrm{a}$ and $1 \mathrm{f}$ with silyl chloride $2 \mathrm{e}$ in different solvents

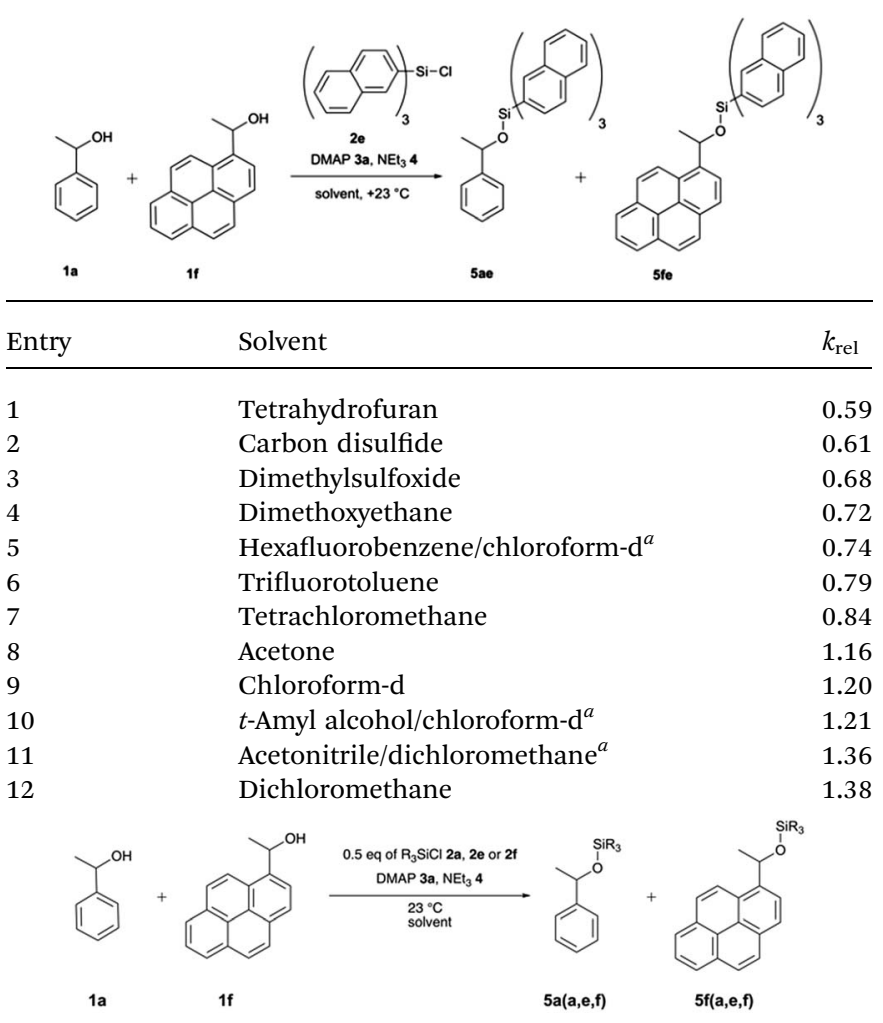

${ }^{a}$ Mixture $1: 1(\mathrm{v} / \mathrm{v})$.

scaled down towards the results obtained for the trialkylsilyl chlorides 2a and $2 \mathbf{b}$ (Fig. 2). The spatial disposition of the substituents in the crystal structure of product $\mathbf{5} \mathbf{f c}$ shows no direct interaction between the naphthyl and pyrenyl surfaces (see ESI $\dagger$ ). Assuming a similar structure in the transition state, relative rates seem to be influenced by the isopropyl as well as the naphthyl substituents. The results presented in Fig. 2 can also be analysed from the point of view of each reacting alcohol (see Fig. S2 $\dagger$ ). While the 9-anthracenyl alcohol 1d containing two peri hydrogen atoms is for all silyl chlorides much slower than 1a, the sterically not hindered alcohols $1 \mathrm{c}$ and $1 \mathrm{e}$ react with all silyl reagents 2 equally fast or faster than 1a. In the 1-pyrenyland 1-naphthyl-substituted alcohols (1f and $\mathbf{1 b}$ ) the relative rate constants are determined by a balance between interactions of the two aromatic surfaces and repulsive steric effects, the former one being dominant in the case of $\mathrm{TN}^{*} \mathrm{SCl} 2 \mathrm{2f}$. In all of the pairs $k_{\text {rel }}$ increases with the growth of the DED-substituent at the silicon centre, which confirms that the size of interacting aromatic surfaces located at the alcohol and silyl substrates determine the chemoselectivity of the silylation reaction.

With the purpose of quantifying the influence of the reaction medium on the relative rate constants, the competition experiment between the reference alcohol 1a and the biggest alcohol 1f with TNSCl 2e was carried out in different solvents. This choice was motivated by two main considerations: (1) both 
alcohol 1f and TNSCl 2e carry the biggest non-substituted DEDsubstituents and (2) using alcohol 1f the balance between attractive aromatic interactions and repulsive steric effects can be studied in different solvents. The $k_{\text {rel }}$ values measured in different solvents span a range from 0.59 in tetrahydrofuran to 1.38 in dichloromethane (Table 1, for details see ESI $\dagger$ ). Strikingly, relative rates for the reaction of alcohol 1f with the naphthyl-substituted silyl chloride $2 \mathrm{e}$ were found to be in several solvents (entries 1-3) very similar to $k_{\text {rel }}$ of the reaction of this alcohol $1 \mathbf{f}$ in the reference solvent $\mathrm{CDCl}_{3}$ with TMSCl $2 \mathrm{a}$ in which no aromatic interactions between alcohol and silyl moiety occur. Therefore, those solvents seem to cancel aromatic interactions almost completely and repulsive steric effects solely govern the relative rates. In contrast, $k_{\text {rel }}$ for the silylation of alcohol $\mathbf{1 f}$ increases up to 2.3 times in other solvents like acetone, chloroform and dichloromethane. In order to prove that those solvent effects are causally related to aromatic interactions, reactions between alcohols $\mathbf{1 a}$ and $\mathbf{1 f}$ with silyl reagents of various sizes were explored by competition experiments in $\mathrm{CDCl}_{3}$ as the reference solvent, and in tetrahydrofuran and dichloromethane as the solvents with the smallest and largest $k_{\text {rel }}$ values in Table 1 (Fig. 3). For TMSCl (2a) as the smallest reagent, only a negligible solvent sensitivity of $k_{\text {rel }}$ was found (Fig. 3), while for the largest reagent $\mathrm{TN}^{*} \mathrm{SCl} 2 \mathrm{2f}$ an increase in solvent sensitivity as compared to the relatively smaller TNSCl $2 \mathrm{e}$ is observed. Hence, the observed solvent effects are due to the significant impact of solvents on sizedependent effects, which was also reported in other studies. ${ }^{26,27,43-46}$ Distinguishing the different contributions of polarizability ( $\pi^{*}$ and $\delta$ ), hydrogen-bond donor $(\alpha)$ and acceptor ability $(\beta)$ via the linear solvation energy relationship developed by Kamlet and $\mathrm{Taft}^{\mathbf{4 7 , 4 8}}$ revealed that solvent effects are widely independent of the polarizability of the solvent, but correlate strongly with the hydrogen bond donor ability of the solvent (see eqn (S12) of ESI $\dagger$ ). This can actually be further condensed to a direct correlation of the experimental $k_{\text {rel }}$ values with the general $\alpha$ parameter proposed by Hunter (Fig. 4). ${ }^{17}$ Considering that the hydrogen-bond donor ability of aromatic $\mathrm{C}-\mathrm{H}$ groups is commonly found to be in the range of $\alpha=1.0-1.4$ the origin of solvent effects can be clarified. Thus, for solvents with $\alpha<1$
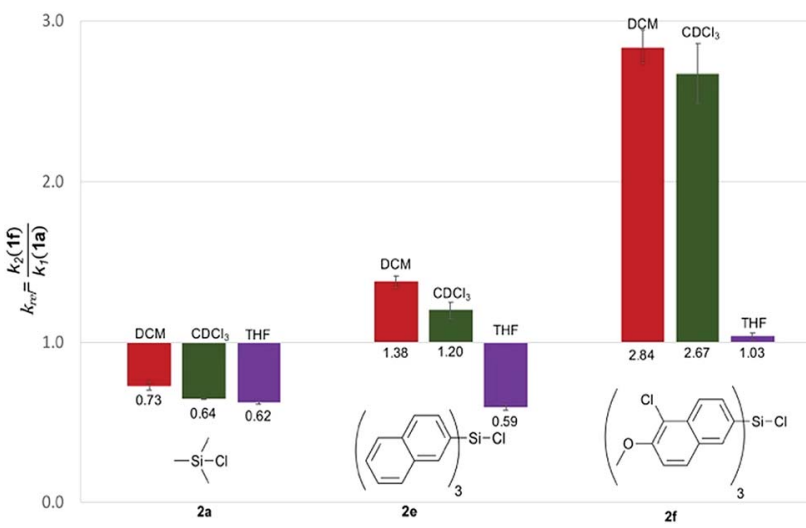

Fig. 3 Relative rate constants $k_{\text {rel }}$ for the reaction of alcohols $1 \mathrm{a}$ and $1 \mathrm{f}$ with silyl chlorides $2 \mathrm{a}, 2 \mathrm{e}$ and $2 \mathrm{f}$ in different solvents.

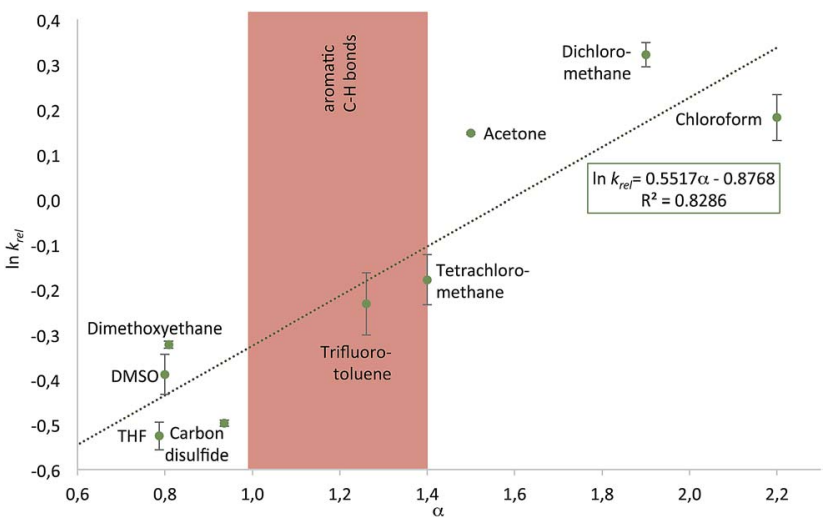

Fig. 4 Plot of experimental $\ln k_{\text {rel }}$ for the reaction of alcohols $1 \mathrm{a}$ and $1 \mathrm{f}$ with silyl chloride $2 \mathrm{e}$ in different solvents against the solvent hydrogen bond donor parameter $\alpha$ defined by Hunter. ${ }^{17}$

such as THF and $\mathrm{CS}_{2}$ hydrogen bonds of aromatic C-H-bonds with solvent molecules dominate the system whereas interactions of the aromatic surfaces of the alcohol and silyl moieties are minimized. Therefore, reaction rates are barely influenced by the different size of the aromatic systems of the two competing alcohols. As $\mathrm{H}$-bonds between the aromatic $\mathrm{C}-\mathrm{H}$ bonds and the solvent get less relevant in solvents with $\alpha>1$ such as chloroform and dichloromethane, desolvation of the alcohol and silyl substrates occurs and as a consequence solvent-solvent as well as aromatic solute-solute interactions become more dominant. ${ }^{17}$ Both the solvophobic effect of solvent molecules forming additional hydrogen bonds among each other and the attractive dispersion forces between the DED-groups can then enhance the rate of the reaction dependent on the size of the aromatic surfaces.

Differentiating the contributions of the aforementioned two types of effects is one main focus of the ongoing debate on aromatic stacking. The $\ln k_{\text {rel }}$ determined in different solvents listed in Table 1 were therefore also analysed in terms of the solvent cohesive energy density (ced) as key parameter for the strength of the solvophobic effect of a solvent. ${ }^{27}$ Whether a higher ced value leads to an increase or a decrease of relative rates appears to depend on the hydrogen bond donor ability of the solvent (Fig. S14 of ESI $\dagger$ ): in solvents with a low $\alpha$ (e.g. THF) higher ced values lead to a reduction in relative rates, possibly through the reinforcement of unfavourable solvent-solute interactions. In contrast, for solvents with a higher $\mathrm{H}$-bond donor ability (e.g. DCM) higher ced values lead to an increase in $k_{\text {rel }}$. Correlations are, however, not very strong in both cases and the ced is thus insufficient to explain the observed differences in $k_{\text {rel }}$. The influence of London dispersion interactions on the experiment shown in Table 1 was subsequently probed through selectivity measurements in $\mathrm{CDCl}_{3}$ at different temperatures, as these interactions are known to be less temperature dependent than dipole-dominated interactions. ${ }^{11}$ Measurements in the temperature range from $-10{ }^{\circ} \mathrm{C}$ to $+23{ }^{\circ} \mathrm{C}$ lead to similar $k_{\text {rel }}$ values for the $\mathbf{1 a} / \mathbf{1 f}$ substrate pair, but the accuracy of these measurements was not high enough for the reliable extraction of activation parameters (see ESI†). 
In how far the relative reaction rates measured experimentally simply reflect the stability of the silyl ether products formed was explored by the calculation of reaction free energies in chloroform solution. Geometry optimizations were performed at the $\operatorname{SMD}\left(\mathrm{CHCl}_{3}\right) / \mathrm{B} 3 \mathrm{LYP}-\mathrm{D} 3 / 6-311+\mathrm{G}(2 \mathrm{~d}) / 6$ $31+\mathrm{G}(\mathrm{d})^{49-53}$ level of theory, followed by single point energy calculations at DLPNO-CCSD(T)/def2-TZVPP ${ }^{54-56}$ level. Solvation free energies were obtained from single point calculations with the $\mathrm{SMD}\left(\mathrm{CHCl}_{3}\right) / \mathrm{B} 3 \mathrm{LYP}-\mathrm{D} 3 / 6-311+\mathrm{G}(2 \mathrm{~d}) / 6-31+\mathrm{G}(\mathrm{d})$ model and added to the gas phase results in order to obtain the reaction free energies in solution $\Delta G_{298 \text {,sol }}$ compiled in Fig. 5 (see ESI $\dagger$ for details). Focusing on the results obtained for pyrenylsubstituted alcohol 1f, we find small and positive reaction energies for the smaller silyl chloride reagents. The positive sign for the reaction energy seen here derives from the fact that the calculated energies exclude the acid/base reaction between $\mathrm{HCl}$ and the auxiliary base $\mathrm{NEt}_{3}$. This is in full agreement with experimental results showing basically no turnover between TBDMSCl (2b) and secondary alcohols in the absence of the auxiliary base. ${ }^{39,40}$ Reaction energies become more favourable and eventually also negative on increasing the size of the silyl chloride reagent. Interestingly, the tert-butyldimethylsilyl ether $\mathbf{5 f b}$ is less stable than the trimethylsilyl ether $\mathbf{5 f a}$, most likely due to repulsive steric interactions between the tert-butyl and the pyrenyl substituents. Although the two interacting aromatic surfaces are the same in the products $\mathbf{5} \mathbf{f c}$ and $\mathbf{5 f e}$, the last one is $18.2 \mathrm{~kJ} \mathrm{~mol}^{-1}$ more stable than $\mathbf{5 f c}$. This energetic difference is possibly associated to the smaller polarizability of the isopropyl substituents than the naphthyl moiety at the Si atom in $\mathbf{5 f c}$. Regarding those results no significant correlations were found between the experimental $k_{\text {rel }}$ and the differences between the $\Delta G_{298, \text { sol }}$ of the respective silyl ethers (see ESI $\dagger$ ), which indicates that the $k_{\text {rel }}$ are purely kinetic phenomena. At this point, we were interested in computing the contribution of the dispersion component to the thermochemical stability of the products. Single point energy calculations were therefore performed at the B3LYP level lacking the D3 dispersion correction over the optimized structures at B3LYP-D3 level (third group, Fig. 5). It was found that $\Delta G_{298 \text {,sol }}$ decreases dramatically (larger positive values) even indicating that these products would be thermodynamically unstable. The smallest dispersion contributions were found in the silyl ethers $\mathbf{5} \mathbf{f a}$ and $\mathbf{5} \mathbf{f b}$ with non-polarizable methyl and tert-butyl substituents. However, in the case of silyl ethers carrying bigger aromatic substituents at the $\mathrm{Si}$ centre, the dispersion component increases notably up to $-85.7 \mathrm{~kJ} \mathrm{~mol}^{-1}$ (5ff). Conformational analysis of silyl ether $\mathbf{5 f e}$ as the silyl ether with the largest unsubstituted aromatic substituents reveals that aromatic surfaces for the best conformers are slightly twisted toward each other so that most non-covalent interactions ${ }^{57}$ arise between the interacting $\pi$ surfaces with a small contribution of $\sigma-\pi$ interactions (see Fig. 5 and ESI $\dagger$ for full details). Interestingly, linear correlations appear to exist between experimental $k_{\text {rel }}$ values and differences in dispersion contributions between the respective substrate pairs $\Delta \Delta D_{298, \text { sol }}$, grouped by the number of 1,5 -interactions at the alcohol substrate (Fig. 6). The similar slope reveals that sizeable DED-groups (higher $\Delta \Delta D_{298, \text { sol }}$ ) increases $\ln k_{\text {rel }}$ equally in both alcohol groups by 0.2 units per $10 \mathrm{~kJ} \mathrm{~mol}^{-1}$ of additional dispersion contribution. That the data points for the unhindered alcohols 1c and 1e fall onto the same correlation line implies that it is irrelevant for the increase of $k_{\text {rel }}$ whether the

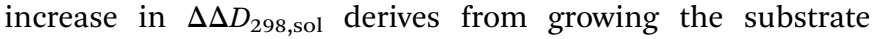
alcohol or the silyl chloride reagent. The presence of one repulsive 1,5-interaction reduces the relative rate by 1.6 times

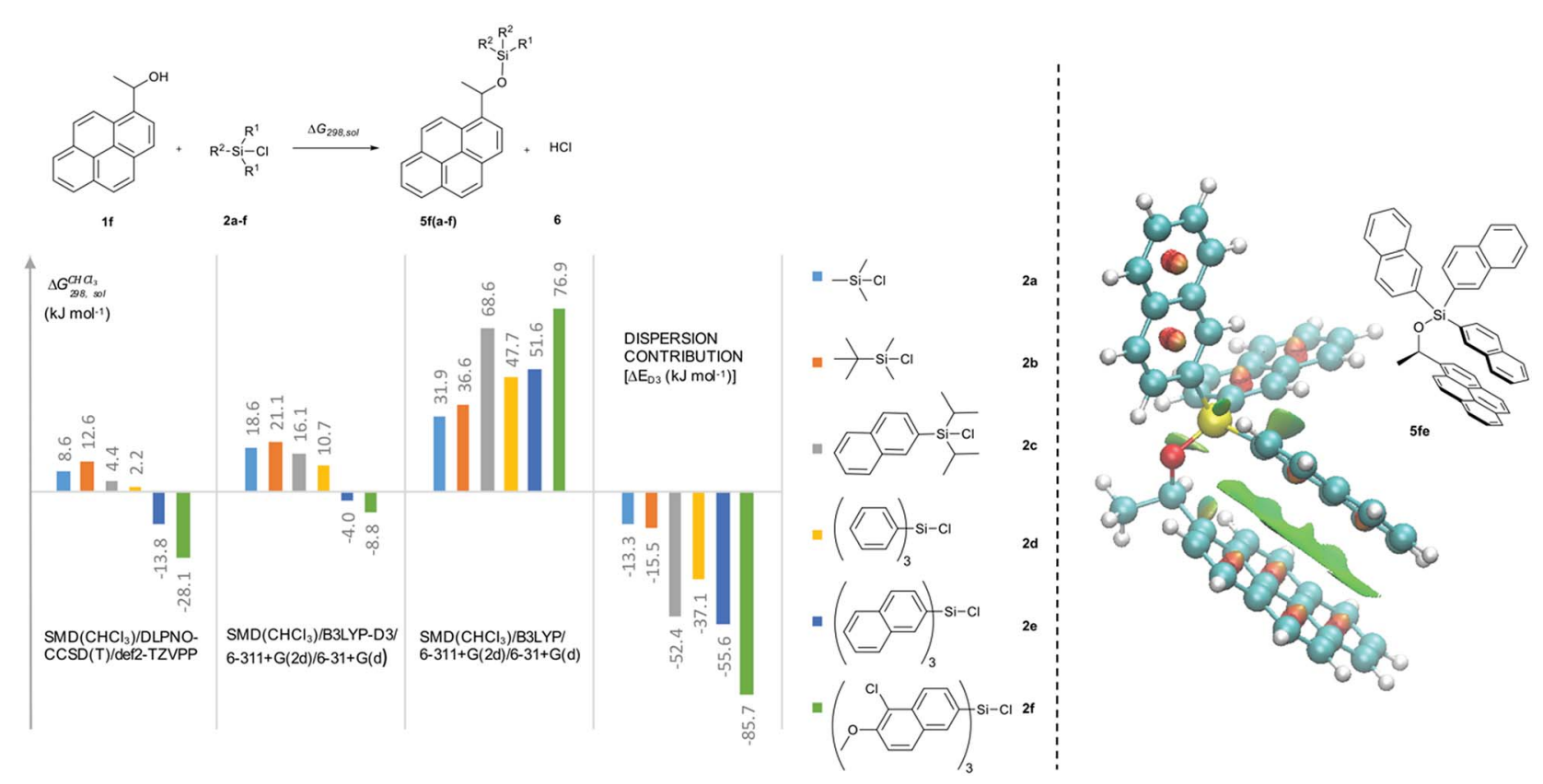

Fig. 5 Reaction free energies $\left(\mathrm{kJ} \mathrm{mol}^{-1}\right)$ for the reaction of $1 \mathrm{f}$ with the silyl reagent $2 \mathrm{a}-\mathrm{f}$ at different levels of theory. Computed non-covalent interaction surfaces (green) of the silyl ether $5 \mathrm{fe}$. 


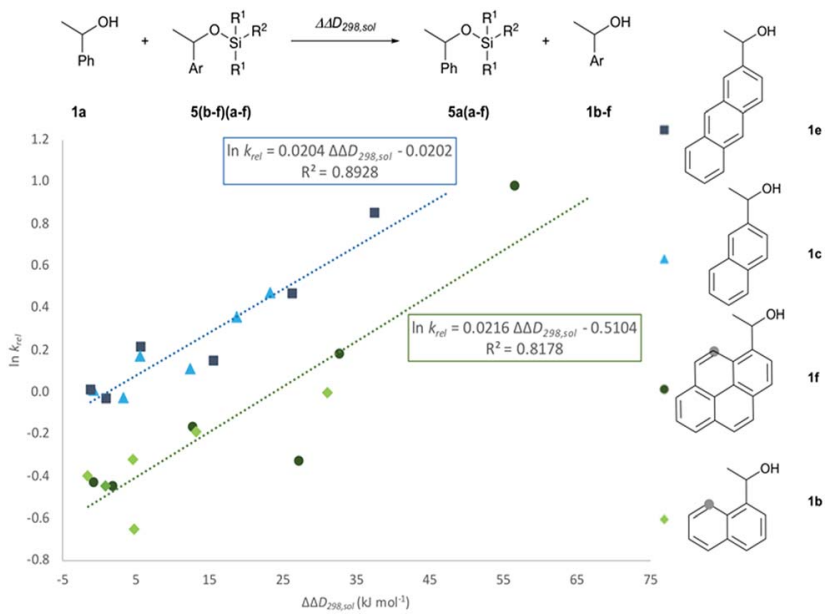

Fig. 6 Correlation of $\ln k_{\text {rel }}$ with calculated differences in the dispersion energy contributions, $\Delta \Delta D_{298, \text { sol. }}$

with respect to the non-hindered alcohols represented by the gap between the two correlation lines in Fig. 6. This analysis demonstrates that the experimentally measured relative rate constants $k_{\text {rel }}$ directly relate to the size of the interacting surfaces and to repulsive steric effects in the alcohol substrates, whereby the dispersion energy component increases together with substrate size.

\section{Conclusions}

In summary, we have experimentally determined relative rates between two secondary alcohols bearing sizeable aromatic surfaces in silylation experiments designed as $1: 1$ competition experiments. In experiments with the comparatively small silyl chloride reagents TMSCl and TBDMSCl the relative rate constants are exclusively governed by repulsive steric effects provoked by the peri hydrogen atoms of the alcohol substrates. However, $k_{\text {rel }}$ increases with the size of the DED groups at the silyl reagent, and aromatic interactions eventually dominate the silylation reactions with reagents as large as $\mathrm{TN}^{*} \mathrm{SCl}$. No significant impact of the reaction temperature on $k_{\text {rel }}$ has been found. In contrast, $k_{\text {rel }}$ depends notably on the solvent used in the competition experiments. While size effects of the interacting aromatic surfaces appear to be cancelled in solvents with poor hydrogen bond donor abilities like tetrahydrofuran, they magnify as solvent-solute interactions get less important in halogenated solvents such as chloroform or dichloromethane depending to a notable extent on the higher solvophobic effect. Computed reaction free energies for the formation of silyl ether products predict that the dispersion component plays a key role in their thermochemical stability. Furthermore, linear correlations were found between experimental $k_{\text {rel }}$ values and the dispersion contribution to the silyl ether formation energy. Therefore, the interplay of attractive dispersion forces and the solvophobic effect enhances relative rates for the silylation of a secondary alcohol up to 4.5 times. In this sense, the combination of sterically less hindered alcohols, tailor-made silyl chloride reagents with bigger DED groups and thoroughly chosen solvents could enhance rate constants even further than in the systems presented here.

\section{Conflicts of interest}

There are no conflicts to declare.

\section{Acknowledgements}

This work was financially supported by the Deutsche Forschungsgemeinschaft (DFG) through the Priority Program "Control of London Dispersion Interactions in Molecular Chemistry” (SPP 1807), grant ZI 436/17-1. Marta Marin-Luna thanks Xunta de Galicia for her postdoctoral contract (ED481B 2016/166-0). We also want to thank the reviewers for their insightful comments and suggestions.

\section{Notes and references}

1 C. A. Hunter, K. R. Lawson, J. Perkins and C. J. Urch, J. Chem. Soc., Perkin Trans. 2, 2001, 5, 651-669.

2 E. H. Krenske and K. N. Houk, Acc. Chem. Res., 2013, 46, 979989.

3 J. K. Klosterman, Y. Yamauchi and M. Fujita, Chem. Soc. Rev., 2009, 38, 1714-1725.

4 F. Biedermann and H.-J. Schneider, Chem. Rev., 2016, 116, 5216-5230.

5 A. Lamouroux, L. Sebaoun, B. Wicher, B. Kauffmann, Y. Ferrand, V. Maurizot and I. Huc, J. Am. Chem. Soc., 2017, 139, 14668-14675.

6 C. Hunter, Chem. Soc. Rev., 1994, 23, 101-109.

7 M. L. Waters, Biopolymers, 2004, 76, 435-445.

8 J. W. Hwang, P. Li and K. D. Shimizu, Org. Biomol. Chem., 2017, 15, 1554-1564.

9 C. R. Martinez and B. L. Iverson, Chem. Sci., 2012, 3, 21912201.

10 S. E. Wheeler, Acc. Chem. Res., 2013, 46, 1029-1038.

11 J. P. Wagner and P. R. Schreiner, Angew. Chem., Int. Ed., 2015, 54, 12274-12296.

12 S. Grimme, R. Huenerbein and S. Ehrlich, ChemPhysChem, 2011, 12, 1258-1261.

13 S. Grimme and P. R. Schreiner, Angew. Chem., Int. Ed., 2011, 50, 12639-12642.

14 S. Rösel, C. Balestrieri and P. R. Schreiner, Chem. Sci., 2017, 8, 405-410.

15 J. N. Israelachvilli, Contemp. Phys., 1974, 15, 159-178.

16 Y. Zhao and J. S. Moore, in Foldamers: Structure, Properties, and Applications, ed. S. Hecht and I. Huc, Wiley-VCH Verlag GmbH \& Co. KGaA, 2007, ch. 3, pp. 75-108.

17 C. A. Hunter, Angew. Chem., Int. Ed., 2004, 43, 5310-5324.

18 S. Otto, Chem. Sci., 2013, 4, 2953-2959.

19 F. Cozzi, F. Ponzini, R. Annunziata, M. Cinquini and J. S. Siegel, Angew. Chem., Int. Ed. Engl., 1995, 34, 1019-1020.

20 S. L. Cockroft, C. A. Hunter, K. R. Lawson, J. Perkins and C. J. Urch, J. Am. Chem. Soc., 2005, 127, 8594-8595. 
21 S. L. Cockroft, J. Perkins, C. Zonta, H. Adams, S. E. Spey, C. M. R. Low, J. G. Vinter, K. R. Lawson, C. J. Urch and C. A. Hunter, Org. Biomol. Chem., 2007, 5, 1062-1080.

22 S. E. Wheeler and K. N. Houk, J. Am. Chem. Soc., 2008, 130, 10854-10855.

23 J. Hwang, P. Li, W. R. Carroll, M. D. Smith, P. J. Pellechia and K. D. Shimizu, J. Am. Chem. Soc., 2014, 136, 14060-14067.

24 H. Gardarsson, W. B. Schweizer, N. Trapp and F. Diederich, Chem.-Eur. J., 2014, 20, 4608-4616.

25 H.-J. Schneider, Acc. Chem. Res., 2015, 48, 1815-1822.

26 L. Yang, C. Adam, G. S. Nichol and S. L. Cockroft, Nat. Chem., 2013, 5, 1006-1010.

27 L. Yang, C. Adam and S. L. Cockroft, J. Am. Chem. Soc., 2015, 137, 10084-10087.

28 C. Adam, L. Yang and S. L. Cockroft, Angew. Chem., Int. Ed., 2015, 54, 1164-1167.

29 L. Yang, J. B. Brazier, T. A. Hubbard, D. M. Rogers and S. L. Cockroft, Angew. Chem., Int. Ed., 2016, 55, 912-916.

30 J. Hwang, B. E. Dial, P. Li, M. E. Kozik, M. D. Smith and K. D. Shimizu, Chem. Sci., 2015, 6, 4358-4364.

31 J. Hwang, P. Li, M. D. Smith and K. D. Shimizu, Angew. Chem., Int. Ed., 2016, 55, 8086-8089.

32 B. Bhayana and C. S. Wilcox, Angew. Chem., Int. Ed., 2007, 46, 6833-6836.

33 R. Pollice, M. Bolt, I. J. Kobylianskii, I. Shenderovich and P. Chen, J. Am. Chem. Soc., 2017, 139, 13126-13140.

34 E. H. Krenske, K. N. Houk and M. Harmata, Org. Lett., 2010, 12, 444-447.

35 J. Helberg, M. Marin-Luna and H. Zipse, Synthesis, 2017, 49, 3460-3470.

36 E. Procházková, A. Kolmer, J. Ilgen, M. Schwab, L. Kaltschnee, M. Fredersdorf, V. Schmidts, R. C. Wende, P. R. Schreiner and C. M. Thiele, Angew. Chem., Int. Ed., 2016, 55, 15754-15759.

37 T. W. Green and P. G. M. Wuts, in Protective Groups in Organic Synthesis, John Wiley \& Sons, Inc, 3rd edn, 2002.

38 P. J. Kocienski, Protecting Groups, Thieme, Stuttgart, 3rd edn, 2005.
39 P. Patschinski and H. Zipse, Org. Lett., 2015, 17, 3318-3321. 40 P. Patschinski, C. Zhang and H. Zipse, J. Org. Chem., 2014, 79, 8348-8357.

41 M. Marin-Luna, P. Patschinski and H. Zipse, Chem.-Eur. J., 2018, submitted.

42 R. K. Akhani, M. I. Moore, J. G. Pribyl and S. L. Wiskur, J. Org. Chem., 2014, 79, 2384-2396.

43 M. S. Cubberley and B. L. Iverson, J. Am. Chem. Soc., 2001, 123, 7560-7563.

44 S. L. Cockroft and C. A. Hunter, Chem. Commun., 2006, 0, 3806-3808.

45 B. U. Emenike, S. N. Bey, B. C. Bigelow and S. V. S. Chakravartula, Chem. Sci., 2016, 7, 1401-1407.

46 A. S. Rury, C. Ferry, J. R. Hunt, M. Lee, D. Mondal, S. M. O. O'Connell, E. N. H. Phan, Z. Peng, P. Pokhilko, D. Sylvinson, Y. Zhou and C. H. Mak, J. Phys. Chem. C, 2016, 120, 23858-23869.

47 M. J. Kamlet, J. M. Abboud and R. W. Taft, J. Am. Chem. Soc., 1977, 99, 6027-6038.

48 M. J. Kamlet, J. M. Abboud, M. H. Abraham and R. W. Taft, J. Org. Chem., 1983, 48, 2877-2887.

49 S. Y. Park, J.-W. Lee and C. E. Song, Nat. Commun., 2015, 6, 7512.

50 A. D. Becke, J. Chem. Phys., 1993, 98, 5648-5652.

51 W. Y. C. Lee and R. G. Parr, Phys. Rev. B, 1988, 37, 785-789. 52 S. Grimme, J. Chem. Phys., 2006, 124, 034108.

53 A. V. Marenich, C. J. Cramer and D. G. Truhlar, J. Phys. Chem. $B$, 2009, 113, 6378-6396.

54 C. Riplinger and F. Neese, J. Chem. Phys., 2013, 138, 034106. 55 C. Riplinger, B. Sandhoefer, A. Hansen and F. Neese, J. Chem. Phys., 2013, 139, 134101.

56 F. Weigend and R. Ahlrichs, Phys. Chem. Chem. Phys., 2005, 7, 3297-3305.

57 J. Contreras-Garcia, E. R. Johnson, S. Keinan, R. Chaudret, J.-P. Piquemal, D. N. Beratan and W. Yang, J. Chem. Theory Comput., 2011, 7, 625-632. 